\title{
Thermoelastic damping in micro- and nanomechanical systems
}

\author{
Ron Lifshitz* and M. L. Roukes \\ Condensed Matter Physics 114-36, California Institute of Technology, Pasadena, California 91125
}

(Received 20 September 1999)

\begin{abstract}
The importance of thermoelastic damping as a fundamental dissipation mechanism for small-scale mechanical resonators is evaluated in light of recent efforts to design high- $Q$ micrometer- and nanometer-scale electromechanical systems. The equations of linear thermoelasticity are used to give a simple derivation for thermoelastic damping of small flexural vibrations in thin beams. It is shown that Zener's well-known approximation by a Lorentzian with a single thermal relaxation time slightly deviates from the exact expression.
\end{abstract}

\section{INTRODUCTION}

Microelectromechanical systems (MEMS) and more recently nanoelectromechanical systems (NEMS) are being developed aggressively for a variety of applications as well as for accessing new regimes of basic experimental research. Among the different applications envisioned for MEMS and NEMS are ultrafast and high-precision actuators, sensors (such as accelerometers, bolometers, magnetometers, and calorimeters), and narrowband high-frequency mechanical filters, all with compact and low-power designs that can be fully integrated with modern semiconductor electronics. Experimentally, it is hoped that NEMS will open the door to the investigation of new regimes of phonon-mediated processes as well as the quantum behavior of mesoscopic mechanical systems. $^{1,2}$

For all these pursuits it is desired to design and construct systems with very little loss of energy or very high quality factors $Q$. Unfortunately, it has been consistently observed that the quality factors of resonators decrease with size significantly-even when made from pure single-crystal materials. It is therefore of great importance to understand the dominating energy dissipation mechanisms in mechanical resonators when one approaches submicron scales. Within these mechanisms one would like to identify those that are fundamental and always impose an upper limit on the quality factor and those that might be eliminated through improved design and fabrication.

In this work we examine the importance of the process of thermoelastic damping as a dissipation mechanism in MEMS and NEMS. Although there has been some discussion of different dissipation mechanisms in MEMS, ${ }^{3-9}$ very few authors have addressed the question of thermoelastic damping. Roszhart $^{3}$ observed thermoelastic damping in single-crystal silicon microresonators at room temperature; and Yasumura et al. ${ }^{9}$ recently reported thermoelastic damping in microresonators an order of magnitude smaller than Roszhart's in silicon nitride, also at room temperature. The question arises whether one should be surprised by such observations or whether one should expect to see thermoelastic damping at these length scales. We establish in this paper that as long as the system remains in the regime of "diffusive thermal phonons" (to be discussed below) the latter is the case for two basic reasons:

(1) The strength of the damping caused by thermoelastic coupling is independent of geometry. It depends only on the thermodynamic properties of the material as a function of temperature.

(2) In the case of flexural vibrations of thin beams the position of peak damping as a function of frequency depends on the dimensions of the beam. Therefore, even though the normal frequencies of the resonators increase as they become smaller so does the frequency at which peak damping occurs.

These effects conspire together to maintain the relevance of thermoelastic damping all the way down to the nanometer scale. In the next section we describe the process of thermoelastic damping, review some of the relevant literature, and present the outline of this paper.

\section{THE PROCESS OF THERMOELASTIC DAMPING}

Acoustic modes - such as a sound wave traveling through an infinitely large elastic material or a normal mode of vibration of an elastic resonator of finite geometry-will experience damping due to their nonlinear interaction with a surrounding bath of thermally-excited elastic modes, or phonons. If the mean free path of these thermal phonons is much smaller than the wavelength of the acoustic mode, then sufficient thermalization occurs on the scale of interest. It is then possible to define a temperature locally, even when the system is not in a state of thermal equilibrium. Equivalently, if the relaxation rate of the phonon distribution to a local Bose-Einstein distribution is much faster than the frequency of the acoustic mode, then one has a well-defined temperature field, and there is no need to treat the thermal phonons as individual excitations. In this regime, in which thermal phonons are said to be "diffusive," the complicated interaction between the acoustic mode and the thermal phonon bath in an isotropic solid is captured by a single macroscopic parameter-the material's thermal-expansion coefficient

$$
\alpha=\frac{1}{L} \frac{\partial L}{\partial T},
$$

which couples changes of length with changes of temperature. Note that here and throughout the paper we make use of the linear coefficient of thermal expansion whose value is one third of the volumetric coefficient of thermal expansion.

When an elastic solid is set in motion, it is taken out of equilibrium, having an excess of kinetic and potential en- 
ergy. In an isothermal and perfectly linear elastic solid such a nonequilibrium state can exist forever. In a thermoelastic solid the coupling of the strain field to a temperature field provides an energy dissipation mechanism that allows the system to relax back to equilibrium. Relaxation of the thermoelastic solid is achieved through the irreversible flow of heat driven by local temperature gradients that through the coupling accompany the strain field. This process of energy dissipation, called thermoelastic damping, is a fundamental one. As long as $\alpha$-which acts as a coupling constant-is nonzero, thermoelastic damping introduces an upper limit to the quality factor of even the most perfectly designed and constructed resonator.

To our knowledge, the first to realize that thermoelastic relaxation may be a significant source of damping in mechanical resonators was Zener, who in a series of papers ${ }^{10}$ in the 1930's developed a general theory of thermoelastic damping. Zener treated the problem in the framework of his so-called "standard model" of the anelastic solid ${ }^{11}$ and showed that the damping behavior can be approximated very well by a single relaxation peak with a characteristic relaxation time. This relaxation time corresponds to the thermal diffusion time across the width $b$ of the beam, which is proportional to $b^{2} / \chi$, where $\chi$ is the solid's thermal diffusivity [to be defined in Eq. (13)]. We shall review Zener's theory in Sec. III.

It was only two decades later that other researchers began reexamining the question of thermoelastic damping by seeking exact solutions to the coupled equations of linear thermoelasticity in various geometries. The solutions for propagating plane waves in an infinite thermoelastic solid ${ }^{12-15}$ showed that the two transverse modes, which propagate through the solid without causing any local volume changes, do not couple to the temperature field and hence suffer no damping. The longitudinal mode, on the other hand, does couple to the temperature field and an exact expression has been obtained for its attenuation and dispersion. Solutions for thermoelastic Rayleigh waves (two-dimensional surface waves on a semi-infinite solid) immediately followed, ${ }^{16}$ but progress on thermoelastic solids with finite geometries came much later due to the well-known difficulty of solving even the equations of linear elasticity with finite boundary conditions. ${ }^{17}$ Nevertheless, analytic solutions now exist, at least in terms of the dispersion relations, for thermoelastic waves in an infinite thin plate, ${ }^{18}$ and longitudinal waves in infinite rods with circular cross sections. ${ }^{15,19,20}$ Other geometries, such as beams of rectangular cross sections, have been too difficult to solve analytically. To treat such problems, one generally needs to use approximate theories.

MEMS and NEMS resonators generally contain elements that vibrate in either torsional or flexural modes. Because of the way one fabricates such devices, rectangular cross sections often turn out to be the most relevant. Pure torsional modes of rectangular beams involve no local volume changes, and therefore, just as for transverse waves in the bulk, they do not suffer any thermoelastic losses. For flexural vibrations of thin rectangular beams, one may use Zener's ${ }^{10}$ approximate expression [Eq. (8)] for thermoelastic damping. This appears to be the general practice. ${ }^{3,9}$ On the other hand, one may try to seek exact solutions of the thermoelastic equations for the case of a thin beam under flexure. Landau and Lifshitz ${ }^{21}$ have provided an exact expression for the attenuation coefficient of such vibrations without, however, giving a rigorous derivation and solution of the equations. Shieh $^{22}$ investigated the thermoelastic beam equations in a more general context while performing a dynamic instability analysis of vertically standing cantilevers. He solved the equations, giving a plot of thermoelastic damping $Q^{-1}$ in thin rectangular beams, but fell short of providing an analytic expression for $Q^{-1}$.

To remedy this state of affairs, we give, in Sec. IV, a simple derivation of the approximate thermoelastic equations for a thin beam under flexure and then solve these equations in Sec. V to arrive at an exact expression [Eq. (29)] for thermoelastic damping in thin rectangular beams. Although Zener's approximation is good for many situations, we suggest that in the future the exact expression given here be used instead. In Sec. VI we discuss the experimental implication of our results showing the characteristic damping curves expected for GaAs and silicon, which are typical materials used in the fabrication of micrometer- and nanometer-scale resonators. In Sec. VII we say a few words about thermoelastic damping of longitudinal waves in MEMS and NEMS, and in Sec. VIII we conclude by discussing the validity of the theory that we present here. In the Appendix we give a careful analysis comparing the exact expression for thermoelastic damping in thin beams with the approximate Lorentzian behavior, predicted by Zener.

\section{ZENER'S STANDARD MODEL OF THE LINEAR ANELASTIC SOLID}

Zener's standard model of anelasticity ${ }^{11}$ is based on an extension of Hooke's law to the most general linear homogeneous equation involving stress $\sigma$, strain $\epsilon$, and their first time derivatives,

$$
\sigma+\tau_{\epsilon} \dot{\sigma}=M_{R}\left(\epsilon+\tau_{\sigma} \dot{\epsilon}\right) .
$$

The physical interpretation of the three parameters of the model is straightforward. When the strain is kept constant, the stress relaxes exponentially with a relaxation time $\tau_{\epsilon}$. Similarly, $\tau_{\sigma}$ is the strain relaxation time when the stress is kept constant. $M_{R}$ is the value of the pertinent elastic modulus after all relaxation has occurred. $M_{U}=M_{R}\left(\tau_{\sigma} / \tau_{\epsilon}\right)$ is the unrelaxed value of the elastic modulus.

Under periodic dynamical conditions

$$
\sigma(t)=\sigma_{0} e^{i \omega t} \quad \text { and } \epsilon(t)=\epsilon_{0} e^{i \omega t},
$$

the stress and strain amplitudes are related by a frequencydependent complex elastic modulus. The dissipation, or "internal friction," in the solid $Q^{-1}$ is defined as the fraction of energy lost per radian of vibration. If it is small, the dissipation is equal to the ratio of the imaginary and real parts of the complex modulus, giving

$$
Q^{-1}=\Delta_{M} \frac{\omega \tau}{1+(\omega \tau)^{2}},
$$

where $\tau=\sqrt{\tau_{\sigma} \tau_{\epsilon}}$, and 


$$
\Delta_{M}=\frac{M_{U}-M_{R}}{\sqrt{M_{R} M_{U}}}
$$

is a dimensionless quantity called the "relaxation strength" of the modulus.

Thus the dissipation exhibits a Lorentzian behavior as a function of $\omega \tau$ with a maximum value of $\Delta_{M} / 2$ when $\omega \tau$ $=1$. Dissipation peaks of this form, generally called "Debye peaks," are quite ubiquitous as to be expected from their prediction by such a naive model. They occur for many different relaxation mechanisms such as point-defect relaxation ("'Snoek peaks"), defect pair reorientation ("Zener peaks"), dislocation relaxation ("Bordoni peaks"), grain-boundary relaxation, and of course thermal relaxation. ${ }^{23}$ In many of these examples there is not just one single relaxation time $\tau$, and therefore one sees multiple or broadened Debye peaks.

One can understand qualitatively why there is a peak in dissipation when $\omega \tau=1$ in the following way: If the frequency of vibration $\omega$ is much smaller than the effective relaxation rate $1 / \tau$ of the solid, then the system remains essentially in equilibrium and very little energy is dissipated. If the vibration frequency is much larger than the effective relaxation rate $\omega \gg 1 / \tau$, the system has no time to relax and again very little energy is dissipated. It is only when the vibration frequency is on the order of the system's effective relaxation rate that appreciable dissipation occurs. The full picture may be more complicated, however, because in some cases $\tau$ itself can depend on $\omega$.

In the case of a thermoelastic solid the relaxation strength (5) to be considered is that of Young's modulus

$$
\Delta_{E}=\frac{E_{\mathrm{ad}}-E}{E}=\frac{E \alpha^{2} T_{0}}{C_{p}}
$$

whose value is known from basic thermodynamics. Here $E_{\text {ad }}$ is the unrelaxed, or adiabatic, value of Young's modulus and $E$ is its relaxed, or isothermal, value. $C_{p}$ is the heat capacity per unit volume at constant pressure, or stress, but replacing it by the heat capacity $C_{v}$ at constant volume, or strain, will introduce an error in $\Delta_{E}$, which is only on the order of $\Delta_{E}^{2}$. Since $Q^{-1}$, and therefore also $\Delta_{E}$, are assumed small, such an error is negligible. For similar considerations, no harm is done by replacing $\sqrt{E_{\mathrm{ad}} E}$ in the denominator of Eq. (5) by $E$.

Zener $^{10,11}$ calculated the thermal relaxation times associated with different transverse thermal modes for a thin beam under flexure. He showed that for rectangular beams approximately $98.6 \%$ of the relaxation occurs through the first mode whose relaxation time is

$$
\tau_{Z}=\frac{b^{2}}{\pi^{2} \chi},
$$

where $\chi$ is the thermal diffusivity of the solid, and $b$ is the width of the beam. Only a very small error is therefore made by considering the vibrating thin beam as having a simple relaxation (4) with a single relaxation time $\tau_{Z}$,

$$
Q_{Z}^{-1}=\frac{E \alpha^{2} T_{0}}{C_{p}} \frac{\omega \tau_{Z}}{1+\left(\omega \tau_{Z}\right)^{2}} .
$$

Nevertheless, we show below that for the simple geometry of a rectangular beam-which is the most relevant for many current MEMS and NEMS designs-such an expansion in transverse thermal eigenmodes is unnecessary, and an exact expression for thermoelastic damping can easily be obtained.

\section{THERMOELASTIC EQUATIONS OF A THIN BEAM}

We consider small flexural displacements of a thin elastic beam of length $L$ and rectangular cross section of dimensions $b \times c$. We define the $x$ axis along the axis of the beam and the $y$ and $z$ axes parallel to the surfaces of dimensions $b$ and $c$, respectively. In equilibrium, the beam is unstrained, unstressed, and at temperature $T_{0}$ everywhere. Departure of the beam from equilibrium is described by a displacement field $u_{i}(i=x, y, z)$ and a temperature field $T=T_{0}+\theta$. The displacement field $u_{i}$ and the relative temperature field $\theta$, as well as the strain and stress tensors $u_{i j}$ and $\sigma_{i j}$, are all functions of position and time.

We consider pure transverse motion $Y(x)$ in the $y$ direction and make the usual Euler-Bernoulli assumption that the transverse dimensions of the beam, $b$ and $c$, are sufficiently small compared with the length $L$ of the beam and the radius of curvature $R$ of the bending that any plane cross section, initially perpendicular to the axis of the beam, remains plane and perpendicular to the neutral surface during bending. The neutral surface is the one running through the length of the beam that suffers no extension or contraction during its bending.

We take the surfaces of the beam to be stress free, which implies that all but the $\sigma_{x x}$ component of the stress tensor vanish on the surface. Because the beam is thin, this approximately holds in its interior as well. Hooke's law for the thermoelastic beam then takes a rather simple form:

$$
\begin{gathered}
u_{x x}=\frac{1}{E} \sigma_{x x}+\alpha \theta, \\
u_{y y}=u_{z z}=-\frac{\sigma}{E} \sigma_{x x}+\alpha \theta, \\
u_{x y}=u_{y z}=u_{z x}=0,
\end{gathered}
$$

where $E$ is Young's modulus, $\sigma$ is Poisson's ratio, $\alpha$ is the linear thermal-expansion coefficient (1), and we have taken into account the fact that strain arises both from mechanical stress as well as thermal expansion. It is simple to show (see, for example, Landau and Lifshitz ${ }^{21}$ ) that the longitudinal strain component $u_{x x}$, a distance $y$ away from the neutral surface, is equal to $y / R$. By replacing the curvature of the beam $1 / R$ with $-\partial^{2} Y / \partial x^{2}$, we may express the nonzero components of the strain field in the beam as

$$
u_{x x}=-y \frac{\partial^{2} Y}{\partial x^{2}}
$$

$$
u_{y y}=u_{z z}=\sigma y \frac{\partial^{2} Y}{\partial x^{2}}+(1+\sigma) \alpha \theta .
$$


Following the standard derivation procedure for isothermal beams with no thermoelastic coupling ${ }^{21}$ but with the modified thermoelastic strain (10) leads to an equation of motion for the beam of the form

$$
\rho A \frac{\partial^{2} Y}{\partial t^{2}}+\frac{\partial^{2}}{\partial x^{2}}\left(E I \frac{\partial^{2} Y}{\partial x^{2}}+E \alpha I_{T}\right)=0
$$

where $\rho$ is the density of the beam, and $A=b c$ is the area of its cross section. The quantities $I$ and $I_{T}$ are integrals over the cross section of the beam giving the mechanical and the thermal contributions to its moment of inertia,

$$
I=\int_{A} y^{2} d y d z=\frac{b^{3} c}{12} \quad \text { and } I_{T}=\int_{A} y \theta d y d z
$$

In evaluating the moment of inertia $I$, we have neglected the deviation of the cross section from its rectangular shape, which arises from having a nonzero Poisson ratio $\sigma$. Such an approximation is justified for small deflections since the error it introduces is only on the order of the transverse beam dimension divided by the radius of curvature of the bending.

To the equation of motion (11) we add the heat equation, which in the presence of thermoelastic coupling is given by ${ }^{21}$

$$
\frac{\partial \theta}{\partial t}=\chi \nabla^{2} \theta-\frac{E \alpha T}{(1-2 \sigma) C_{v}} \frac{\partial}{\partial t} \sum_{j} u_{j j} .
$$

We make two simplifications to this equation. First, since $\theta$ $\ll T_{0}$, we can safely replace $T$ by $T_{0}$ in the second term on the right-hand side of the equation. Not doing so will introduce unnecessary nonlinearities into the problem. Second, noting that thermal gradients in the plane of the cross section along the $y$ direction are much larger than gradients along the beam axis and that no gradients exist in the $z$ direction, we replace $\nabla^{2} \theta$ with $\partial^{2} \theta / \partial y^{2}$. Substituting the value of the strain field from Eq. (10), we finally get

$$
\left(1+2 \Delta_{E} \frac{1+\sigma}{1-2 \sigma}\right) \frac{\partial \theta}{\partial t}=\chi \frac{\partial^{2} \theta}{\partial y^{2}}+y \frac{\Delta_{E}}{\alpha} \frac{\partial}{\partial t} \frac{\partial^{2} Y}{\partial x^{2}},
$$

where we have identified the relaxation strength of Young's modulus $\Delta_{E}$ [Eq. (6)].

\section{SOLUTION OF THE THERMOELASTIC EQUATIONS FOR HARMONIC VIBRATIONS}

To calculate the effect of thermoelastic coupling on the vibrations of a thin beam, we solve the coupled thermoelastic equations (11) and (14) for the case of harmonic vibrations. We set

$$
Y(x, t)=Y_{0}(x) e^{i \omega t}, \quad \text { and } \theta(x, y, t)=\theta_{0}(x, y) e^{i \omega t},
$$

calculate the temperature profile along the beam's cross section using the heat equation (14), and substitute it into the equation of motion (11) in order to obtain the normal modes of vibration and their corresponding frequencies. We expect to find that in general the frequencies are complex, the real part $\operatorname{Re}(\omega)$ giving the new eigenfrequencies of the beam in the presence of thermoelastic coupling, and the imaginary part $|\operatorname{Im}(\omega)|$ giving the attenuation of the vibration. The amount of thermoelastic damping, expressed in terms of the inverse of the quality factor, will then be given by

$$
Q^{-1}=2\left|\frac{\operatorname{Im}(\omega)}{\operatorname{Re}(\omega)}\right|,
$$

which is the fraction of energy lost per radian, the factor of 2 arising from the fact that the mechanical energy of the beam is proportional to the square of its amplitude.

Substituting Eq. (15) into the heat equation (14) and neglecting the term of order $\Delta_{E}$ on its left-hand side (which will only introduce a correction of order $\Delta_{E}^{2}$ to the final result) yields the following equation for $\theta_{0}$ :

$$
\frac{\partial^{2} \theta_{0}}{\partial y^{2}}=i \frac{\omega}{\chi}\left(\theta_{0}-\frac{\Delta_{E}}{\alpha} \frac{\partial^{2} Y_{0}}{\partial x^{2}} y\right),
$$

whose solution is

$$
\theta_{0}-\frac{\Delta_{E}}{\alpha} \frac{\partial^{2} Y_{0}}{\partial x^{2}} y=A \sin (k y)+B \cos (k y),
$$

where

$$
k=\sqrt{i \frac{\omega}{\chi}}=(1+i) \sqrt{\frac{\omega}{2 \chi}} .
$$

The coefficients $A$ and $B$ are determined by taking as boundary conditions the requirement that there be no flow of heat across the boundaries of the beam so that $\partial \theta_{0} / \partial y=0$ at $y$ $= \pm b / 2$. The temperature profile across the beam is then given by

$$
\theta_{0}(x, y)=\frac{\Delta_{E}}{\alpha} \frac{\partial^{2} Y_{0}(x)}{\partial x^{2}}\left(y-\frac{\sin (k y)}{k \cos \left(\frac{b k}{2}\right)}\right)
$$

Now that we have the temperature profile we can substitute it into the integral $I_{T}$ [Eq. (12)] for the cross section's thermal moment. Because $E, I$, and $\Delta_{E}=E \alpha^{2} T_{0} / C$ are all constant along the beam, the beam equation may subsequently be expressed as

$$
\omega^{2} Y_{0}=\frac{E I}{\rho A}\left\{1+\Delta_{E}[1+f(\omega)]\right\} \frac{\partial^{4} Y_{0}}{\partial x^{4}},
$$

where the complex function $f(\omega)$ is given by

$$
f(\omega)=f(k(\omega))=\frac{24}{b^{3} k^{3}}\left[\frac{b k}{2}-\tan \left(\frac{b k}{2}\right)\right] .
$$

The equation of motion for the beam (21) is formally identical to that of the isothermal beam with no thermoelastic coupling. The only difference being that the isothermal value of Young's modulus $E$ is replaced by a frequency-dependent modulus 


$$
E_{\omega}=E\left\{1+\Delta_{E}[1+f(\omega)]\right\}
$$

When $\omega$ becomes very large, $f(\omega) \rightarrow 0$, and Young's modulus tends to its adiabatic, or unrelaxed, value $E_{a d}=E(1$ $\left.+\Delta_{E}\right)$. When $\omega$ is very small, $f(\omega) \rightarrow-1$, and Young's modulus recovers its isothermal value $E$, as expected. For intermediate frequencies, $E_{\omega}$ is complex.

The normal modes of vibration of the beam are given, as in the isothermal case, by

$Y_{0}(x)=A \sin (q x)+B \cos (q x)+C \sinh (q x)+D \cosh (q x)$,

where the coefficients $A$ through $D$ and the allowed values of $q$ are determined, as usual, by the boundary conditions at the two ends of the beam. For example, for beams clamped at both ends or free at both ends $q_{n} L=a_{n}$ $=\{4.730,7.853,10.996, \ldots\}$, and for cantilevers clamped at one end and free at the other $q_{n} L=a_{n}$ $=\{1.875,4.694,7.855, \ldots\}$, in all three cases tending for large $n$ to odd-integer multiples of $\pi / 2$. The dispersion relation between $\omega$ and $q_{n}$ for the thermoelastic beam is given by

$$
\omega=\sqrt{\frac{E_{\omega} I}{\rho A}} q_{n}^{2}=\omega_{0} \sqrt{1+\Delta_{E}[1+f(\omega)]},
$$

where $\omega_{0}$ is the isothermal value of the eigenfrequency.

Neglecting corrections of order $\Delta_{E}^{2}$, we may replace $f(\omega)$ in the square root by $f\left(\omega_{0}\right)$. The dispersion relation (25) then becomes

$$
\omega=\omega_{0}\left[1+\frac{\Delta_{E}}{2}\left[1+f\left(\omega_{0}\right)\right],\right.
$$

from which we can easily extract the real and imaginary parts, giving the thermoelastic corrections of order $\Delta_{E}$ to the eigenfrequencies of the beam as well as the corresponding attenuation coefficients

$$
\begin{gathered}
\operatorname{Re}(\omega)=\omega_{0}\left[1+\frac{\Delta_{E}}{2}\left(1-\frac{6}{\xi^{3}} \frac{\sinh \xi-\sin \xi}{\cosh \xi+\cos \xi}\right)\right] \\
\operatorname{Im}(\omega)=\omega_{0} \frac{\Delta_{E}}{2}\left(\frac{6}{\xi^{3}} \frac{\sinh \xi+\sin \xi}{\cosh \xi+\cos \xi}-\frac{6}{\xi^{2}}\right)
\end{gathered}
$$

where

$$
\xi=b \sqrt{\frac{\omega_{0}}{2 \chi}} .
$$

The universal behavior of the normalized frequency shift $\left[\operatorname{Re}(\omega)-\omega_{0}\right] / \omega_{0} \Delta_{E}$ and of the normalized attenuation $\operatorname{Im}(\omega) / \omega_{0} \Delta_{E}$ as functions of the dimensionless variable $\xi$ are shown in Fig. 1.

Using the definition (16) of the quality factor, we arrive at an expression for thermoelastic damping in a thin beam, which, to first order in $\Delta_{E}$, is given by

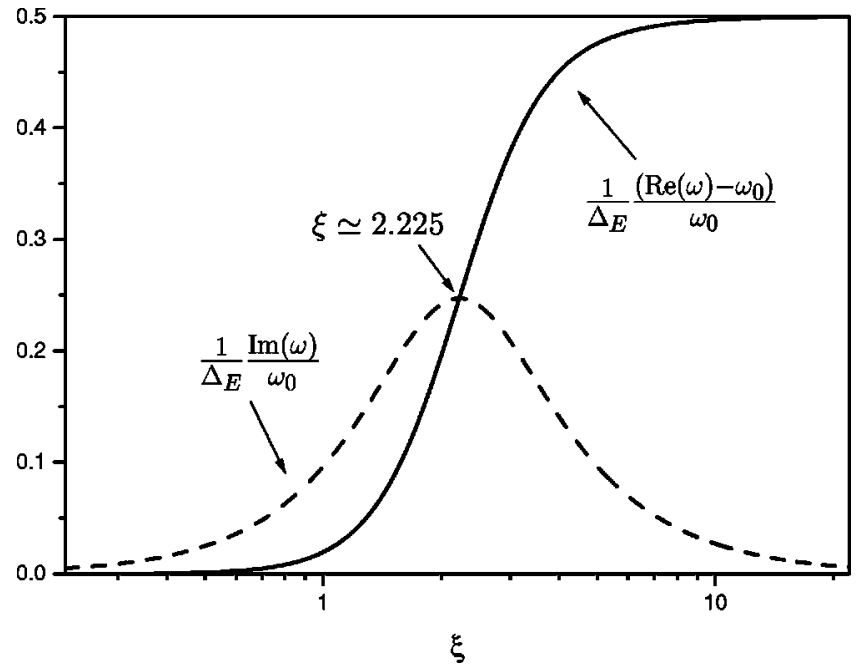

FIG. 1. Universal plots of the frequency shift and attenuation, Eq. (27), of small flexural vibrations in thin rectangular beams due to thermoelastic coupling.

$$
Q^{-1}=\frac{E \alpha^{2} T_{0}}{C}\left(\frac{6}{\xi^{2}}-\frac{6}{\xi^{3}} \frac{\sinh \xi+\sin \xi}{\cosh \xi+\cos \xi}\right)
$$

\section{DISCUSSION OF THE RESULTS AND THEIR EXPERIMENTAL IMPLICATIONS}

We show in the Appendix that the exact expression (29) for thermoelastic damping is tightly bound between two Lorentzians in the variable $\xi^{2} / \sqrt{24}$ and that it behaves as $\xi^{2}$ for small $\xi$ and as $1 / \xi^{2}$ for large values of $\xi$. We also compare the exact result with Zener's approximate expression (8). The damping is peaked at $\xi_{0} \simeq 2.225$ with a maximum value of $Q_{\max }^{-1} / \Delta_{E} \simeq 0.494$. The universal behavior of $Q^{-1 / \Delta_{E}}$ as a function $\xi$ is shown in Fig. 2 .

The first conclusion to be drawn from this universal behavior is that the peak value of thermoelastic damping, given approximately by $0.494 \Delta_{E}$, is independent of the dimensions of the beam. It only depends on temperature through the thermodynamic properties $E, \alpha$, and $C$ of the material. The values of $Q_{\max }^{-1}$ for GaAs and silicon, typical materials used in the fabrication of MEMS and NEMS, are plotted as a function of temperature in Fig. 3. Thus, for example, at room temperature one expects to observe quality factors no higher than $10^{3}-10^{4}$ if one is operating at around $\xi=2.225$.

The dimensionless variable $\xi^{2}$ is proportional to the product $\omega \tau$ used in Zener's model of the anelastic solid. Because we are mainly concerned with the dependence of thermoelastic damping on scale, and since both $\omega$, the beam's eigenfrequency, and $\tau$, its thermal relaxation time, depend on the dimensions of the beam, we find it more suitable to explicitly express the dependence of $\xi$ on the dimensions of the beam. Instead of the usual plots of Debye peaks as a function of frequency, we plot the damping curve as a function of the dimensions of the beam.

To do that, we express the (isothermal) eigenfrequencies (25) in terms of the beam dimensions

$$
\omega^{(n)}=a_{n}^{2} \frac{b}{L^{2}} \sqrt{\frac{E}{12 \rho}} .
$$




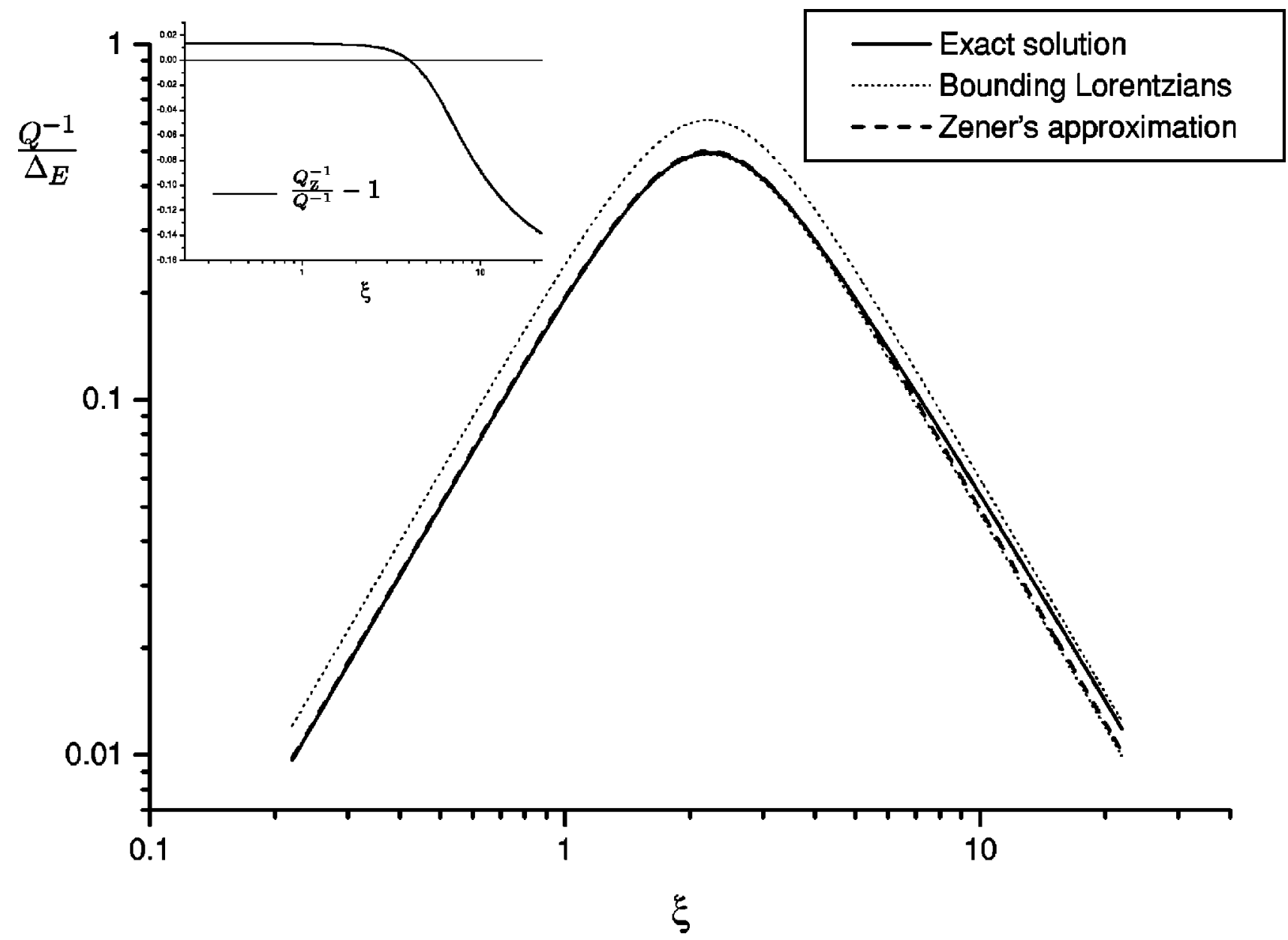

FIG. 2. Universal plot of thermoelastic damping of small flexural vibrations in thin beams (29). The damping is plotted in units of the relaxation strength $\Delta_{E}=E \alpha^{2} T_{0} / C$, as a function of the dimensionless variable $\xi=b \sqrt{\omega_{0} / 2 \chi}$ for one decade above and below its peak value $\simeq 0.494$, occurring at $\xi \simeq 2.225$. The two bounding Lorentzians (A3) in the variable $\xi^{2} / \sqrt{24}$ are shown along with Zener's approximation $Q_{Z}^{-1} / \Delta_{E}$ [Eq. (8)]. The relative error in Zener's approximation $\left(Q_{Z}^{-1}-Q^{-1}\right) / Q^{-1}$ is shown in the inset.

We then get

$$
\xi^{2}=\frac{a_{n}^{2}}{4 \sqrt{3}} \frac{b^{3}}{L^{2} l_{T}},
$$

where $l_{T}$ is a thermal diffusion length, proportional to the phonon mean free path, given by

$$
l_{T}=\chi \sqrt{\frac{\rho}{E}} .
$$

We use experimentally reported values of the thermodynamic properties of GaAs (Ref. 24) and silicon ${ }^{25}$ to obtain $l_{T}$ as well as $\Delta_{E}(T)$, listed in Table I, for three representative temperatures: $10 \mathrm{~K}, 100 \mathrm{~K}$, and $300 \mathrm{~K}$. We then use these values for illustrative purposes to plot the dependence of thermoelastic damping on geometry in three different ways: (1) $Q^{-1}$ vs beam width $b$ for fixed aspect ratio $L / b$, (2) $Q^{-1}$ vs beam width $b$ for fixed beam length $L$, and (3) $Q^{-1}$ vs beam length $L$ for fixed beam width $b$. The outcome is shown in Fig. 4 for the case of a beam clamped at both ends vibrating in its fundamental mode $\left(a_{n}=4.73\right)$. The slopes of the curves, plotted in $\log -\log$ scale, are $\pm 1, \pm 3$, and \pm 2 , respectively, which is easily understood through the relation (31) and the fact that $Q^{-1}$ grows as $\xi^{2}$ for small $\xi$ and decays as $\xi^{-2}$ for large $\xi$.

It is clear from these examples that thermoelastic damping is a significant source of dissipation for MEMS and NEMS at temperatures around $100 \mathrm{~K}$ and above. The reason for this is simple and follows from the fact that as the beam becomes smaller its eigenfrequency increases at the same time that its thermal relaxation time decreases. The product of the two, which can be controlled by independently varying the two dimensions $b$ and $L$, can therefore remain of order unity down to the nanometer scale.

\section{THERMOELASTIC DAMPING OF LONGITUDINAL WAVES}

For completeness we would like to say a few words regarding thermoelastic damping of longitudinal modes. Even though such modes do not come into play when considering MEMS and NEMS resonators they may affect general questions of heat flow and energy relaxation in other elements of MEMS and NEMS. The process of thermoelastic damping of longitudinal waves is similar to that of flexural waves in that energy is dissipated through the irreversible flow of heat 


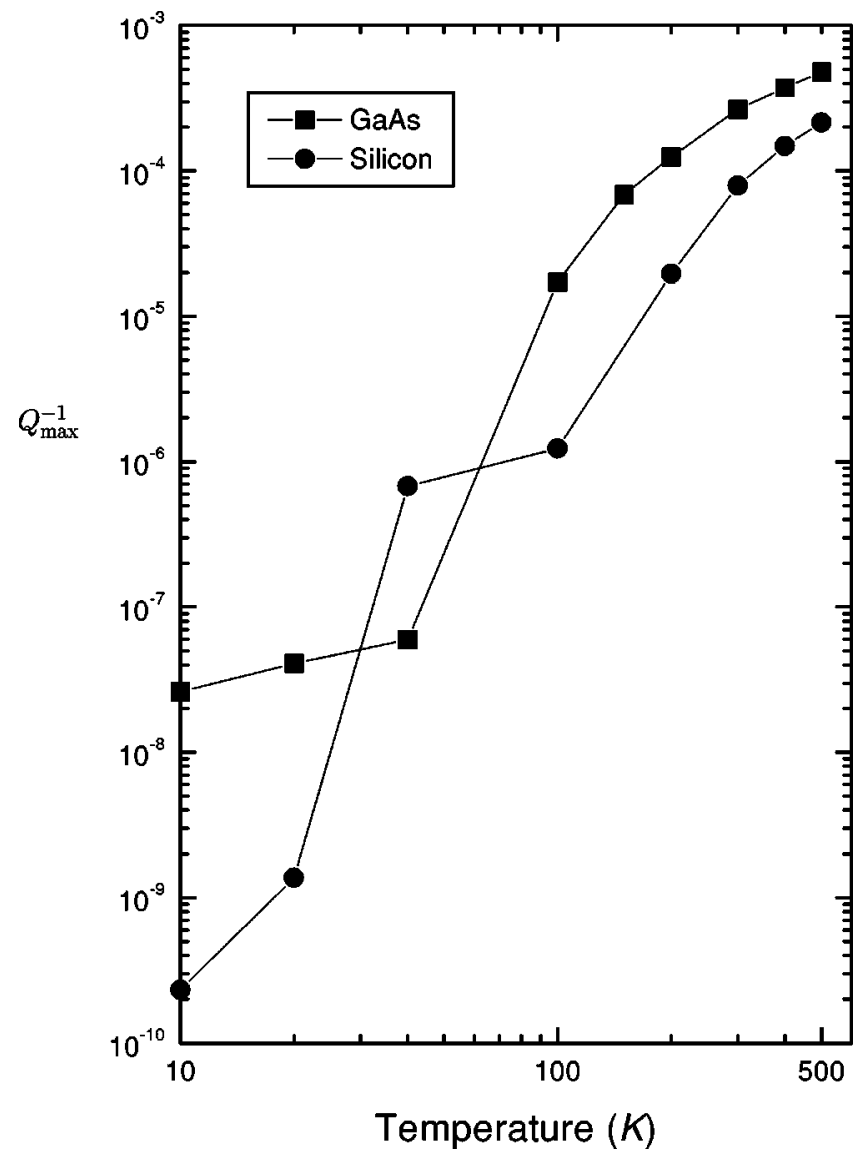

FIG. 3. Peak value of thermoelastic damping $Q_{\max }^{-1} \simeq 0.494 \Delta_{E}$ (maximum value of the plot in Fig. 2) plotted for gallium arsenide and for silicon as a function of temperature. Experimentally reported values (Refs. 24 and 25) are used for the thermodynamic quantities $E, \alpha$, and $C$.

from hot to cold regions of the solid. The difference is that the distance between these regions is not fixed by the transverse geometry of the device. Because a longitudinal wave is a compression wave, hot and cold regions are separated by half a wavelength $\lambda$ along the propagation direction of the wave. The wavelength $\lambda$ is inversely proportional to the frequency, and therefore the thermal relaxation time $\tau_{l}$ for a longitudinal thermoelastic wave, which is proportional to

TABLE I. Relaxation strengths $\Delta_{E}(T)$ and thermal diffusion lengths $l_{T}$ (in $\mu \mathrm{m}$ ) for GaAs and silicon at three representative temperatures. The values are calculated from experimental data (Refs. 24 and 25) and are used for generating the plots of Fig. 4.

GaAs

\begin{tabular}{lccc}
$T$ & $10 \mathrm{~K}$ & $100 \mathrm{~K}$ & $300 \mathrm{~K}$ \\
\hline$\Delta_{E}(T)$ & $2.612 \times 10^{-8}$ & $1.718 \times 10^{-5}$ & $2.651 \times 10^{-4}$ \\
$l_{T}(\mu \mathrm{m})$ & $1.300 \times 10^{+2}$ & $4.456 \times 10^{-2}$ & $6.455 \times 10^{-3}$
\end{tabular}

\begin{tabular}{lccc}
\multicolumn{4}{c}{ Silicon } \\
$T$ & $10 \mathrm{~K}$ & $100 \mathrm{~K}$ & $300 \mathrm{~K}$ \\
\hline$\Delta_{E}(T)$ & $2.319 \times 10^{-10}$ & $1.232 \times 10^{-6}$ & $7.942 \times 10^{-5}$ \\
$l_{T}(\mu \mathrm{m})$ & $4.977 \times 10^{+2}$ & $2.017 \times 10^{-1}$ & $1.257 \times 10^{-2}$ \\
\hline \hline
\end{tabular}

$\lambda^{2} / \chi$, is in fact inversely proportional to the square of the frequency. It is therefore the case that high-frequency waves that have very short thermal relaxation times are isothermal, or relaxed, and low-frequency waves are adiabatic, or unrelaxed. This quite counterintuitive situation is exactly the opposite of what one has in the case of flexural vibrations of beams and is probably the best example of a system in which the relaxation time $\tau$ depends on the frequency $\omega$.

Nevertheless, as can be seen, for example, in the work of Chadwick and Sneddon, ${ }^{14}$ thermoelastic damping of longitudinal waves still takes the form of a relaxation peak, with the isothermal and adiabatic limits exchanged, and with a geometry-independent characteristic thermal relaxation time $\tau_{l}=\chi / c_{l}^{2}$ where $c_{l}$ is the speed, or phase velocity, of longitudinal waves. Thus, the position of peak thermoelastic damping for longitudinal waves is fixed and only depends on the thermodynamic properties of the material as a function of temperature. Some typical values for the thermal relaxation rates of longitudinal waves in GaAs and silicon are given in Table II.

\section{CONCLUSIONS}

We have established here that thermoelastic damping is a significant source of dissipation down to the nanometer scale. We gave a simple derivation of an exact expression (29) for thermoelastic damping in thin rectangular beams, compared this exact expression with Zener's well-known approximation (8), and examined the implication of our result on micrometer- and nanometer-scale resonators. It is interesting to note, as a consequence of our analysis [see Figs. 4(a) and 4(b)], that for beams of constant aspect ratio and constant temperature above a certain beamwidth the quality factor increases linearly with the size of the beam. This may provide a partial explanation for the linear increase in dissipation as systems become smaller.

We have made a number of approximations and assumptions along the way that we would like to summarize here.

(1) We have derived and solved the thermoelastic equations of a thin beam undergoing small flexural vibrations. We should not expect our result to strictly hold for beams with small aspect ratios $L / b$. Nevertheless, we do expect to see the same kind of behavior, showing a Debye-like dissipation peak, even at smaller aspect ratios. We do not expect our result to hold for large amplitude vibrations where the Euler-Bernoulli assumption is known to fail, and where nonlinear behavior begins to take over.

(2) In displaying the expected relaxation strengths (Fig. 3) and damping curves (Fig. 4) for GaAs and silicon resonators with various geometries, we used experimentally-reported bulk elastic and thermodynamic properties. According to recent molecular-dynamics simulations of thin quartz beams by Broughton et $a l .^{26}$ one is justified in using bulk properties down to beamwidths of about $10 \mu \mathrm{m}$. For smaller widths, quantities such as Young's modulus change drastically. In any case, one should consider the plots only as serving for illustrative purposes, in particular because some of the thermodynamic properties, especially below about $200 \mathrm{~K}$, vary from sample to sample.

(3) The theory of thermoelasticity is valid in a regime where thermal phonons are diffusive and a temperature field 


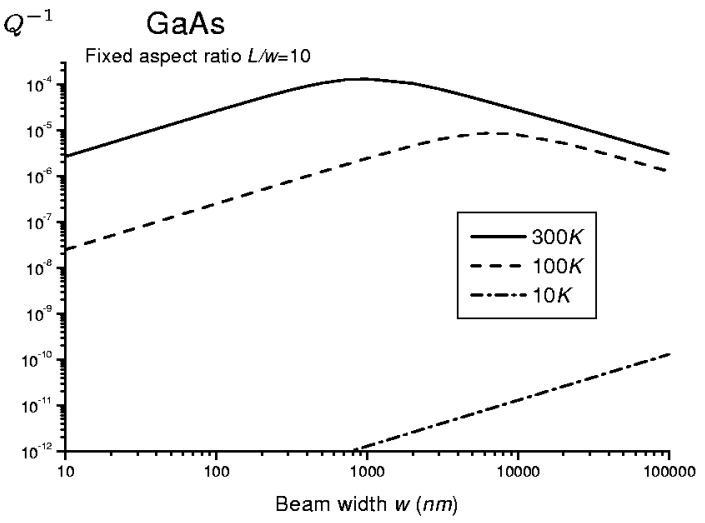

(a)

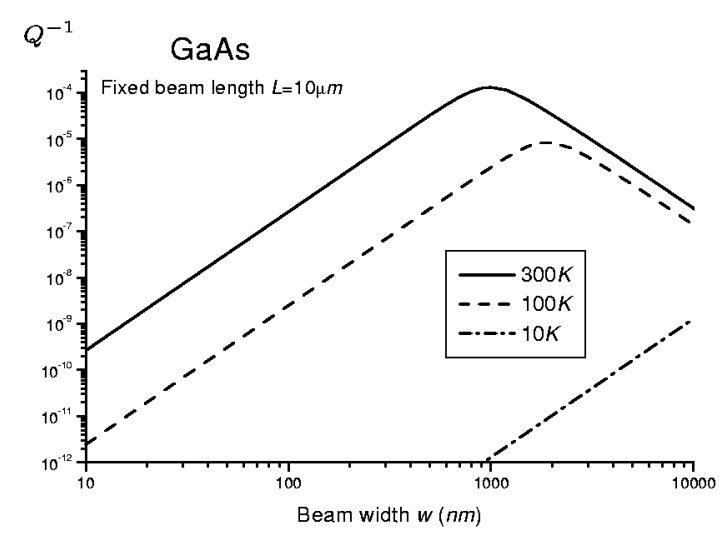

(c)

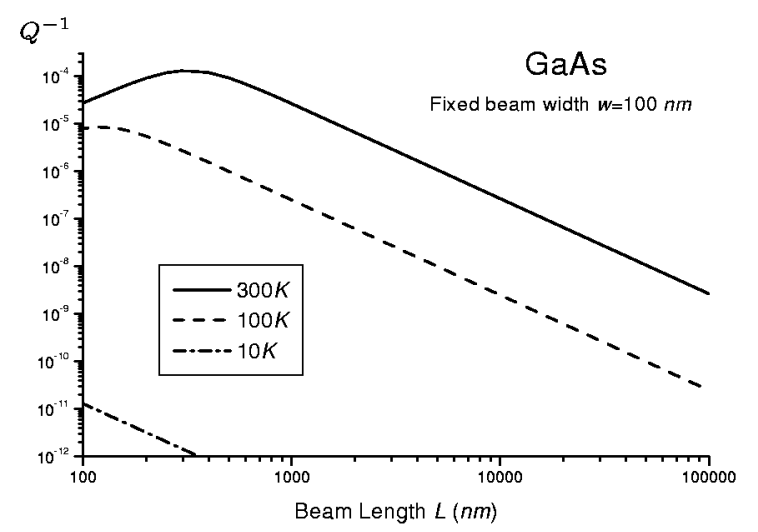

(e)

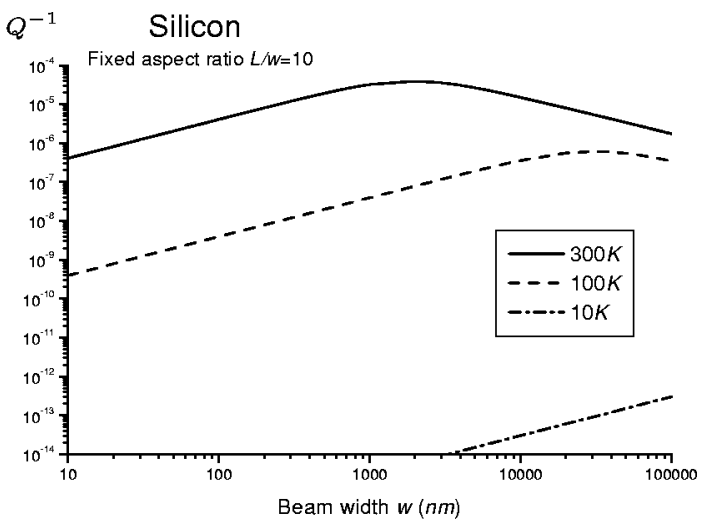

(b)

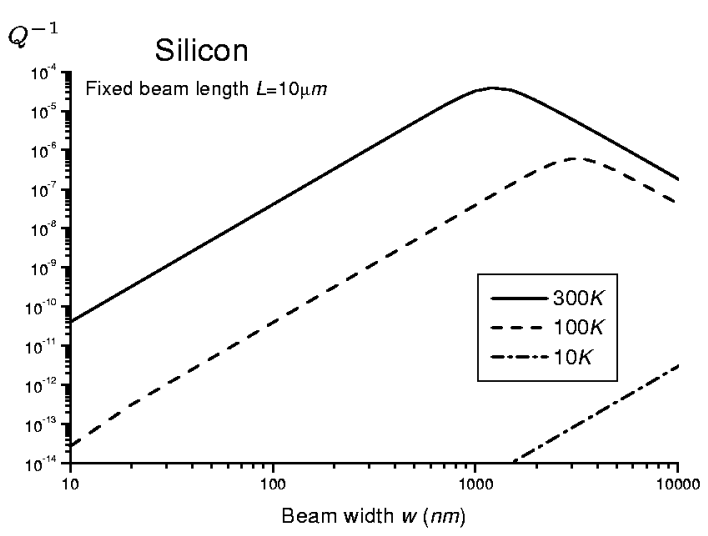

(d)

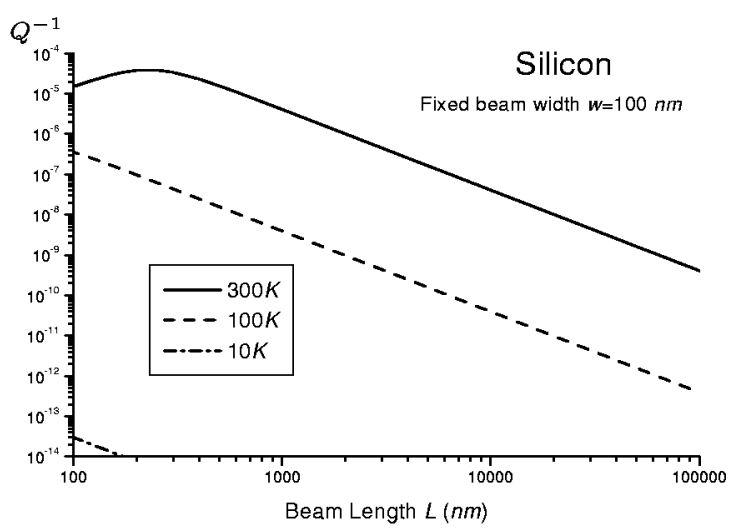

$(f)$

FIG. 4. Thermoelastic damping in gallium arsenide and silicon thin rectangular beams plotted for different geometries. In all cases the beams are assumed clamped at both ends and vibrating at their fundamental flexural mode. The corresponding thermal diffusion lengths $l_{T}$ and relaxation strengths $\Delta_{E}(T)$, used here, are listed in Table I.

can be defined locally. One should expect to see deviations from this theory when the phonon mean free path becomes comparable to the system size, or when the relaxation rate of the phonons to their equilibrium Bose distribution becomes comparable to the resonator's frequency. In the first case, where the transport of thermal energy crosses over from being diffusive to being ballistic, one may expect to see thermal relaxation times that are linear in the beamwidth (proportional to $b / v$ where $v$ is the phonon velocity) instead of the diffusive quadratic dependence $b^{2} / \chi$. The second effect introduces an additional dissipation mechanism that may be viewed as caused by the "viscosity" of the phonon gas as it relaxes to its equilibrium state. This latter mechanism is called the "Akhiezer effect." 27 We intend to explore these corrections to the diffusive regime, as well as the fully ballistic phonon regime, in the context of mesoscopic systems in a future publication.

\section{ACKNOWLEDGMENTS}

We thank Michael Cross for many helpful conversations. We also thank members of our group, Eyal Buks, Kamil 
TABLE II. Thermal relaxation rates $\nu_{l}=1 / 2 \pi \tau_{l}=c_{l}^{2} / 2 \pi \chi$ for longitudinal thermoelastic waves in GaAs and silicon at three representative temperatures. The values are calculated from experimentally reported data (Refs. 24 and 25) and listed here to illustrate the typical frequencies at which thermoelastic damping of longitudinal waves is most significant. The relaxation strengths at these temperatures are the same as those listed in Table I.

\begin{tabular}{lccc}
\hline \hline & \multicolumn{3}{c}{ GaAs } \\
\hline$\nu_{l}$ & $10 \mathrm{~K}$ & $100 \mathrm{~K}$ & $300 \mathrm{~K}$ \\
\hline & $7.1 \mathrm{MHz}$ & $10.0 \mathrm{GHz}$ & $138 \mathrm{GHz}$ \\
\hline$\nu_{l}$ & $10 \mathrm{~K}$ & Silicon & \\
\hline \hline
\end{tabular}

Ekinci, Darrell Harrington, Raj Mohanty, and Keith Schwab, for ongoing discussion and collaboration. We gratefully acknowledge support from DARPA MTO/MEMS under Grant No. DABT63-98-0012.

\section{APPENDIX: WHERE IS THE LORENTZIAN?}

Because Zener's approximation (8) using a single Lorentzian in the variable $\omega \tau_{Z}$ is so good, it is illuminating to try to identify this Lorentzian behavior in our own result (29). To do so, let us expand both numerator and denominator of $Q^{-1 / \Delta_{E}}$ in powers of $\xi$,

$$
\begin{aligned}
\frac{Q^{-1}}{\Delta_{E}}= & 6 \frac{\frac{4}{5 !} \xi^{2}+\frac{8}{9 !} \xi^{6}+\cdots}{1+\frac{1}{4 !} \xi^{4}+\frac{1}{8 !} \xi^{8}+\cdots} \\
= & 6 \frac{\frac{4}{5 !} \xi^{2}\left(1+\frac{2 \cdot 5 !}{9 !} \xi^{4}+\frac{3 \cdot 5 !}{13 !} \xi^{8}+\cdots\right)}{1+\frac{\xi^{4}}{4 !}\left(1+\frac{4 !}{8 !} \xi^{4}+\frac{4 !}{12 !} \xi^{8}+\cdots\right)} .
\end{aligned}
$$

One can clearly see that for small values of $\xi$, the two series in parentheses each tend to 1 , their ratio approaching 1 from above as $\xi$ decreases. For large values of $\xi$, the leading 1 in the denominator may be neglected, and one can show with a little bit of effort that the ratio of the two series in parentheses tends to $5 / 4$ from below as $\xi$ increases. Defining $\mathcal{L}$ as the Lorentzian

$$
\mathcal{L}(\eta)=\frac{\eta}{1+\eta^{2}}
$$

we see that for any value of $\xi, Q^{-1} / \Delta_{E}$ is bounded between the two Lorentzians

$$
\frac{2 \sqrt{6}}{5} \mathcal{L}\left(\frac{\xi^{2}}{\sqrt{24}}\right) \leqslant \frac{Q^{-1}}{\Delta_{E}} \leqslant \frac{\sqrt{6}}{2} \mathcal{L}\left(\frac{\xi^{2}}{\sqrt{24}}\right)
$$

Figure 2 shows the universal thermoelastic damping curve $Q^{-1} / \Delta_{E}$ in relation to these two bounding Lorentzians. The inset shows the difference between Zener's Lorentzian approximation, which in the above notation takes the form

$$
Q_{Z}^{-1}=\Delta_{E} \mathcal{L}\left(\frac{\xi^{2}}{\pi^{2} / 2}\right)
$$

and the exact result (29). Note that $\pi^{2} / 2 \simeq 4.935$ and $\sqrt{24}$ $\simeq 4.899$ differ by less than $1 \%$. It should be emphasized that on the isothermal side of the peak (low frequencies) the two expressions differ by less than $2 \%$, which is the error anticipated by Zener in keeping only the first term in his expansion. On the adiabatic side of the peak (high frequencies), the error increases in the first decade to as much as $15 \%$, reaching $20 \%$ in the limit of infinite $\xi$.
*Present address: School of Physics and Astronomy, Tel Aviv University, Tel Aviv 69978, Israel. Electronic address: ronlif@post.tau.ac.il.

${ }^{1}$ M. L. Roukes, Physica B 263-264, 1 (1999).

${ }^{2}$ A. N. Cleland and M. L. Roukes, in Proceedings of the 24th International Conference on the Physics of Semiconductors (World Scientific, Singapore, in press).

${ }^{3}$ T. V. Roszhart, Proceedings of the Solid-State Sensor and Actuator Workshop, Hilton Head Island, SC, 1990 (IEEE, New York, 1990), p. 13.

${ }^{4}$ F. R. Blom, S. Bouwstra, M. Elwenspoek, and J. H. J. Fluitman, J. Vac. Sci. Technol. B 10, 19 (1992).

${ }^{5}$ R. E. Mihailovich and N. C. MacDonald, Sens. Actuators A 50, 199 (1995)

${ }^{6}$ D. S. Greywall, B. Yurke, P. A. Busch, and S. C. Arney, Europhys. Lett. 34, 37 (1996).

${ }^{7}$ D. W. Carr, L. Sekaric, and H. G. Craighead, J. Vac. Sci. Technol. B 16, 3821 (1998).
${ }^{8}$ K. Y. Yasumura, T. D. Stowe, E. M. Chow, T. Pfafman, T. W. Kenny, and D. Rugar, Proceedings of the Solid-State Sensor and Actuator Workshop, Hilton Head Island, SC, 1998 (IEEE, New York, 1998), p. 65.

${ }^{9}$ K. Y. Yasumura, T. D. Stowe, T. W. Kenny, and D. Rugar, Bull. Am. Phys. Soc. 44(1), 540 (1999).

${ }^{10}$ C. Zener, Phys. Rev. 52, 230 (1937); 53, 90 (1938); C. Zener, W. Otis, and R. Nuckolls, ibid. 53, 100 (1938).

${ }^{11}$ For a review, see C. Zener, Elasticity and Anelasticity of Metals (The University of Chicago Press, Chicago, 1948).

${ }^{12}$ M. A. Biot, J. Appl. Phys. 27, 240 (1956).

${ }^{13}$ H. Deresiewicz, J. Acoust. Soc. Am. 29, 204 (1957).

${ }^{14}$ P. Chadwick and I. N. Sneddon, J. Mech. Phys. Solids 6, 223 (1958).

${ }^{15}$ For a review, see P. Chadwick, in Progress in Solid Mechanics Vol. 1, edited by I. N. Sneddon and R. Hill (North-Holland, Amsterdam, 1960), Chap. VI.

${ }^{16}$ F. J. Lockett, J. Mech. Phys. Solids 7, 71 (1958). 
${ }^{17}$ For a review on elastic waves in bars of finite transverse geometry, see W. A. Green, in Progress in Solid Mechanics Vol. 1 (Ref. 15), Chap. V.

${ }^{18}$ M. Daimaruya and M. Naitoh, J. Sound Vib. 117, 511 (1987).

${ }^{19}$ M. Daimaruya and M. Naitoh, Acustica 51, 124 (1982).

${ }^{20}$ E. S. Suhubi, J. Therm. Stresses 9, 279 (1986).

${ }^{21}$ L. D. Landau and E. M. Lifshitz, Theory of Elasticity (Pergamon Press, Oxford, 1959).

${ }^{22}$ R.-C. Shieh, J. Appl. Mech. 38, 839 (1971). The same author later found a similar expression for thermoelastic damping in circular rods under flexure. See R.-C. Shieh, ibid. 42, 405 (1975).

${ }^{23}$ For a detailed review, see A. S. Nowick and B. S. Berry, Anelastic Relaxation in Crystalline Solids (Academic Press, New York, 1972).

${ }^{24}$ Properties of Gallium Arsenide, edited by M. R. Brozel and G. E. Stillman, 3rd ed. (INSPEC, London, 1996).

${ }^{25}$ Properties of Silicon (INSPEC, London, 1988).

${ }^{26}$ J. Q. Broughton, C. A. Meli, P. Vashista, and R. K. Kalia, Phys. Rev. B 56, 611 (1997).

${ }^{27}$ A. Akhiezer, J. Phys. (Moscow) 1, 277 (1939). 